\title{
Effect of Antarctic Krill Oil on Astaxanthin in Rat Serum
}

\author{
Tang Yixin ${ }^{1}$, Zhang Huizhen ${ }^{2}$
}

\author{
${ }^{1}$ Department of Public Health, Qingdao University School, Qingdao 266071, China \\ ${ }^{2}$ Qingdao Food and Drug Inspection Institute, Qingdao 266071, China
}

\begin{abstract}
To explore whether the antarctic krill oil has an impact on astaxanthin ester absorption process, provide scientific basis and theoretical support for the development and application of Antarctic krill oil in functional food or special medical food. Methods: 150 Wistar male rats were randomly divided into 5 groups: blank control group, $0.208 \mathrm{~g}$ oil $/ \mathrm{kg}$.bw Antarctic krill oil group, $1.25 \mathrm{mg} / \mathrm{kg}$ body wt of astaxanthin group, $1.25 \mathrm{mg} / \mathrm{kg}$ body wt of astaxanthin $+0.208 \mathrm{~g}$ oil $/ \mathrm{kg}$.bw Antarctic krill oil group and $1.25 \mathrm{mg} / \mathrm{kg}$ body wt of astaxanthin $+0.208 \mathrm{~g}$ oil $/ \mathrm{kg}$.bw Fish oil group.Samples were collected at ten time points $(0,0.5,1,2,4,8,10,12,16,24 \mathrm{~h})$ after administering a single oral dose corresponding to rats via the intragastric gavage mode and the astaxanthin concentrations in samples were detected using Hplc.Results: The maximum concentration of astaxanthin in the serum of ASTA- krill oil group was delayed by $6 \mathrm{~h}$ compared with the ASTA group. The highest concentration of ASTA- krill oil group in serum was was 5.3 times of ASTA- Fish oil group, which was 8.4 times that of ASTA group. The serum AUC values of ASTA-krill oil group,the ASTA group and the ASTA-Fish oil group were $108.39 \pm 2.34 \mathrm{ug} \cdot \mathrm{h} / \mathrm{L}, 14.35 \pm 0.21 \mathrm{ug} \cdot \mathrm{h} / \mathrm{L}$, $23.39 \pm 0.46 \mathrm{ug} \cdot \mathrm{h} / \mathrm{L}$, respectively., reflecting that the bioavailability of ASTA- krill oil in rats is higher than that of ASTA group, which is better than ASTA- Fish oil .Conclusion: The serum absorption rate of the ASTA- krill oil group are excellent. Antarctic krill oil promotes the bioavailability of astaxanthin and provides scientific basis and theoretical support for the development and application of functional foods or special medical foods.
\end{abstract}

Keywords: Antarctic Krill Oil, Rat, Astaxanthin, Serum

\section{Introduction}

Antarctic krill (Euphausia superba), belonging to the arthropods (Arthropoda), Crustacea, Euphausiacea, Euphausiidae, Euphausia, living in a cluster The single species of plankton in the Southern Ocean has a huge biomass of about 6.5 to 1 billiontons. The annual catch of biological life is equivalent to the sum of the current world fishery production ${ }^{[1]}$. As a new treasure trove of marine quality protein and active lipids, Antarctic krill has become the most important development target of China's oceanic strategic resources.

At present, Antarctic krill oil is extracted from Antarctic krill by extraction technology. Antarctic krill oil has functions such as improving lipid metabolism, regulating blood pressure, preventing oxidation, improving sleep, preventing and treating neurodegenerative diseases ${ }^{[2]}$.

Astaxanthin is an oxygen-containing derivative of fat-soluble ketone carotenoids, which is a terpene-based unsaturated compound, which can effectively quench active oxygen and scavenge free radicals, and has high nutritional value ${ }^{[3]}$. Astaxanthin is a natural antioxidant, and its antioxidant capacity is much higher than other active substances such as vitamin $\mathrm{E}$ and other carotenoids lutein, lycopene, etc. [4].

At present, ultraviolet spectrophotometry, high performance liquid chromatography and thin layer chromatography are used to quantitatively analyze astaxanthin and astaxanthin esters, among which high performance liquid chromatography is used. Therefore, this experiment used astaxanthin ester extracted from Haematococcus pluvialis as raw material, and compared with Antarctic krill oil and astaxanthin ester by gavage in rats, systematically investigated the intake of Antarctic krill. The distribution and accumulation of astaxanthin in serum, whether the Antarctic krill oil was produced during the digestion and absorption of astaxanthin ester. The impact provides scientific and theoretical support for the development and application of Antarctic krill oil in the field of functional foods or special medical uses.

\section{Materials and methods}

\subsection{Chemicals and reagents}

Antarctic krill oil was obtained from Antarctic Weikang Biotechnology (Qingdao,China),TG fish oil was provided by Gaolong Industrial (Fujian,China). Astaxanthin ester was purchased from Qingdao Algae Biology Co., Ltd., which extracted from 
Haematococcus pluvialis, and the content of astaxanthin was $16 \%$. Pig bile salt was purchased from Shanghai Johnson Biotechnology Co., Ltd.

All-reverse astaxanthin standard (purity 95.8 $\pm 0.5 \%$ ) was purchased from Dr.Ehrenstorfer KGaA (Germany). HPLC-grade methanol and Methyl tert-butyl ether were obtained from Merck KGaA (Darm- stadt, Germany). Acetone, chloroform, methanol, phosphoric acid and other reagents or solvents were commercially available and reagent grade. Ultrapure water was produced by a Milliplus 2150 system (Millipore Corp., Billerica, MA, USA).

\subsection{Liquid chromatography conditions}

Analyte separations were performed on High Performance Liquid Chromatograph DGU-20A5R with UV Detector(Shimadzu Corporation,Japan) using an YMC-Carotenoid C30 column (250 mm×4.6 mm,5um, YMC Corporation, Japan) maintained at $35^{\circ} \mathrm{C}$. The mobile phase was composed of methanol(A), methyl tert-butyl ether(B) and water $(\mathrm{C}, 1 \%$ phosphate)(A:B:C=81:15:4, v/v/v) at a flow rate of $1.0 \mathrm{~mL} / \mathrm{min}$ and the injection volume was $20 \mathrm{uL}$.

\subsection{Standard curves}

Individual standard stock solutions of All-reverse astaxanthin $(0.1 \mathrm{mg} / \mathrm{mL})$ was prepared in acetone. These stock solutions were then serially diluted with acetone to provide standard working solutions in the concentration range of $10-1000 \mathrm{ug} / \mathrm{L}$ for fucoxanthinol. All solutions were stored at $4^{\circ} \mathrm{Cand}$ brought to room temperature before use.

Standard curves were prepared by acetone the appropriate analyte working solution. The effective concentrations were $10,20,30,50,70,80 \mathrm{ug} / \mathrm{L}$ for astaxanthin then divided into aliquots, and stored at $4{ }^{\circ} \mathrm{C}$ until use.

2.4.In vivo animal study for tissue accumulation According to the method of Wang Jiahui ${ }^{[5]}$, according to the experimental group, add a certain amount of astaxanthin and krill oil or fish oil and mix them evenly, then add $0.5 \%$ pig bile salt as emulsifier, vortex and mix and put in water bath. Ultrasound was ultrasonicated, and then homogenized for $5 \mathrm{~min}$ at $10,000 \mathrm{r} / \mathrm{min}$ in a high-speed dispersing homogenizer to form a homogeneous mixed solution, which was then placed in a refrigerator for storage to prepare a uniform fat emulsion for intragastric administration. The volume of the gavage was $0.5 \mathrm{~mL} / 100 \mathrm{~g}$.

The experimental protocol was approved by the Ethics Committee for Animal Experimentation of Qing dao University. Six- week old male Wistar rats were purchased from Shandong Lukang Pharmaceutical Co., Ltd. Experimental Animal Center, production license number: SCXK (Lu) 20140001.All rats were housed individually in cages under specific pathogen-free conditions at a temperature of $20-25^{\circ} \mathrm{C}$, humidity of $40 \% \sim 70 \%$ and maintained on a $12 \mathrm{~h}$ light/dark cycle with continuous access to food and water. After a 1-week adaptation period, all rats were dosed following overnight fasting except for water adli- bitum.

For studies, 150 rats were randomly divided into five groups. Rats in Group 1 were administered with $1.25 \mathrm{mg} / \mathrm{kg}$ body wt of astaxanthin. In Group 2, each rat received the administration with $1.25 \mathrm{mg} / \mathrm{kg}$ body wt of astaxanthin and $0.208 \mathrm{~g}$ oil $/ \mathrm{kg}$.bw Antarctic krill oil. In Group 3, each rat received the administration with $1.25 \mathrm{mg} / \mathrm{kg}$ body wt of astaxanthin and $0.208 \mathrm{~g}$ oil/kg.bw TG fish oil. In Group 4, each rat received the administration with $0.208 \mathrm{~g}$ oil $/ \mathrm{kg}$.bw Antarctic krill oil. In Group 5, each rat received the administration with $1.25 \mathrm{mg} / \mathrm{kg}$ body wt of Saline .

samples were collected at ten time points ( 0 , $0.5,1,2,4,8,10,12,16,24 \mathrm{~h})$ after administering a single oral dose corresponding to rats via the intragastric gavage mode and the astaxanthin concentrations in samples were detected using Hplc. the rats were sacrificed by the abdominal aorta anesthetized with $3 \%$ sodium pentobarbital $(0.3 \mathrm{ml} / 100 \mathrm{~g})$. The serum was separated and stored at $-80^{\circ} \mathrm{C}$ until use.

\section{5. sample preparation}

Serum: To $500 \mathrm{uL}$ of Serum sample in a $10 \mathrm{~mL}$ polypropylene tube, $2.5 \mathrm{~mL}$ of methanol - chloroform $(1: 2, \mathrm{v} / \mathrm{v})$ were added and mixed by vortexing for $60 \mathrm{~s}$. After centrifugation at $8000 \times \mathrm{g}$ for $5 \mathrm{~min}$, the chloroform phase (lower layer) was collected and was filtered through a membrane $(0.45$ um pore size), and evaporated. The residue was dissolved in a mixture of methanol/MBTE (1:1, v/v), and an aliquot was subjected to HPLC analysis within 3 days.

\subsection{Statistical analysis}

All experiments were performed in triplicates. All data were expressed as mean standard deviation. Data were analyzed using one-way ANOVA $(\mathrm{P}<0.05)$. The significant differences among means were separated by Turkey s HSD post hoc test. The one-way ANOVA analyses were performed using Microsoft Office Professional Plus 2007 Excel. Turkey s HSD post hoc test (Confidence interval 95\%) was conducted using the IBM SPSS 17.0 statistical package.

\section{Results and discussion \\ 3.1 Method validation}

3.1.1.Identification of astaxanthin by HPLC

Astaxanthin was identified by HPLC ${ }^{[6]}$. The corresponding gradient elution procedure are shown in Table 1.The total chromatography time was $35.0 \mathrm{~min}$ and retention times for astaxanthin was 10.40 min(Fig.1). Astaxanthin was detected in samples after intragastric administration. The astaxanthin in a rat serum sample at T-2h was shown in the Figs. 2. 
Table 1 Gradient elution procedure of HPLC for astaxanthin

\begin{tabular}{cccc}
\hline Time $(\min )$ & $\begin{array}{c}\text { Mobile phase A } \\
(\%)\end{array}$ & $\begin{array}{c}\text { Mobile phase B } \\
(\%)\end{array}$ & $\begin{array}{c}\text { Mobile phase C } \\
(\%)\end{array}$ \\
\hline 0 & 81 & 15 & 4 \\
15 & 66 & 30 & 4 \\
23 & 16 & 80 & 4 \\
27 & 16 & 80 & 4 \\
30 & 81 & 15 & 4 \\
\hline
\end{tabular}

$\mathrm{mV}$

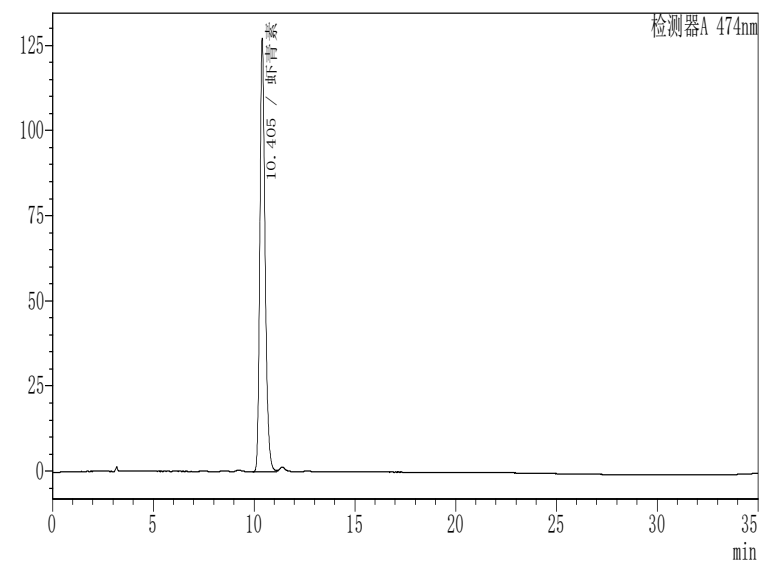

Fig. 1 The HPLC chromatograms of astaxanthin $\mathrm{ml}$

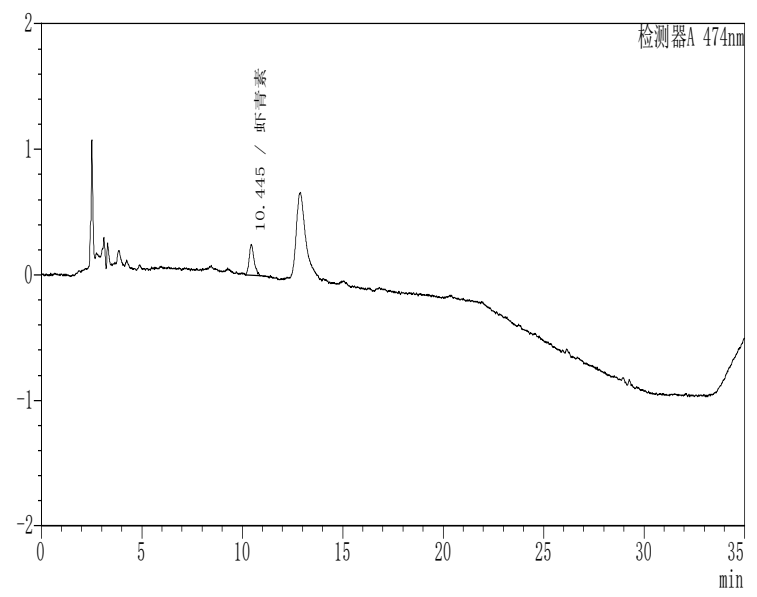

Fig. 2 The HPLC chromatograms of astaxanthin at $\mathrm{T}-2 \mathrm{~h}$ in a rat serum sample
3.1.2. Linearity of the calibration curve, lower limit of quantification (LLOQ), and limit of detection (LOD) Standard curves were established by plotting of chromatogram peak areas of astaxanthin. The curves showed correlation coefficients of $>0.999$ and exhibited good linearity over the concentration range 10-80 ug/L.Typical equations for the calibration curves were $\mathrm{y}=207.371 \mathrm{x}-24.3083 \quad(\mathrm{R}=0.9996)$, where $\mathrm{y}$ represents the peak area of an analyte, and $\mathrm{x}$ represents an analyte concentration. The LLOQ for astaxanthin was $0.97 \mathrm{ug} / \mathrm{L}$ based on a signal-to- noise ratio $(\mathrm{S} / \mathrm{N})$ of 10.1. The LOD was estimated to be $0.32 \mathrm{ug} / \mathrm{L}$ based on an $\mathrm{S} / \mathrm{N}$ of 3.7 .

\subsubsection{Recovery}

The recoveries of astaxanthin spiked into rat serum were determined at three concentrations. The recoveries of astaxanthin were $103.4 \pm 3.70 \%$, $93.2 \pm 9.48 \%$, and $89.3 \pm 2.72 \%(n=3)$ at concentrations of 25,50 , and $250 \mathrm{ug} / \mathrm{L}$, respectively (Table 3 ).

Table 2 Recovery of astaxanthin in serum samples

\begin{tabular}{lll}
\hline Analyte & Concentration(ug/L) & Mean \pm SD(ug/L) \\
\hline \multirow{3}{*}{ Astaxanthin } & 25 & $103.4 \pm 3.70$ \\
& 50 & $93.2 \pm 9.48$ \\
& 250 & $89.3 \pm 2.72$ \\
\hline
\end{tabular}

The recoveries of astaxanthin spiked into rat intestinal contents were determined at three concentrations. The recoveries of astaxanthin were $93.0 \pm 3.48 \%, 105.3 \pm 6.01 \%$, and $88.1 \pm 2.60 \%(n=3)$ at concentrations of 25,50 , and 100ug, respectively (Table 4). 
Table 3 Recovery of astaxanthin in rat intestinal contents samples

\begin{tabular}{ccc}
\hline Analyte & Concentration(ug) & Mean \pm SD(ug/L) \\
\hline \multirow{3}{*}{ Astaxanthin } & 25 & $93.0 \pm 3.48$ \\
& 50 & $105.3 \pm 6.01$ \\
& 100 & $88.1 \pm 2.60$ \\
\hline
\end{tabular}

3.2. pharmacokinetic study in serum

The accumulation rates of carotenoids in animal organ tissues can be directly related to its plasma concentration $^{[7,8]}$. Hence, a pharmacokinetic study was utilized to elucidate the correlation between the tissue accumulation efficiency and the serum absorption rate of astaxanthin according to the food materials.

The pharmacokinetic profiles of astaxanthin were investigated by the described method following a single dose of astaxanthin $(1.25 \mathrm{mg} / \mathrm{kg}$ body weight.)and Antarctic krill oil(0.208g oil $/ \mathrm{kg} . \mathrm{bw})$ to rats. The mean serum concentration-time profiles of astaxanthin are shown in Fig. 4.

As seen in Table 5, among the four tested samples (ASTA, ASTA-Krill Oil, ASTA-Fish Oil and Krill Oil), ASTA-Krill Oil showed a remarkably high area under the serum concentration- time curve (AUCt) value for astaxanthin $(108.39 \pm 2.34 \mathrm{ug} \cdot \mathrm{h} / \mathrm{L})$ when compared to the other three samples where the values ranged from 0 to $23.39 \pm 0.46 \mathrm{ug} \cdot \mathrm{h} / \mathrm{L}$. AUCt values for total astaxanthin were in the following order: ASTA-Krill Oil $(108.39 \pm 2.34 \mathrm{ug} \cdot \mathrm{h} / \mathrm{L}) \quad>\quad$ ASTA-Fish Oil $(23.39 \pm 0.46 \mathrm{ug} \cdot \mathrm{h} / \mathrm{L}) \quad>\quad$ ASTA $(14.35 \pm 0.21 \mathrm{ug} \cdot \mathrm{h} / \mathrm{L})$. These results indicate that the serum absorption of astaxanthin is higher when astaxanthin is ingested with Antarctic krill oil or fish oil, especially, with Antarctic krill oil. Astaxanthin was absorbed slowly and was detected in plasma $0.5 \mathrm{~h}$ after administration and rose slowly thereafter. The lower Tmax values of the ASTA-Krill Oil (6-8h) than that of ASTA (8-10h) for astaxanthin further supports the increased serum absorption rate of astaxanthin through the Antarctic krill oil. From these pharmacokinetic results, we could confirm that the higher accumulation rate of astaxanthin in organ tissues of ASTA-Krill Oil and ASTA-Fish Oil animal groups was induced by the higher serum absorption rate of these Krill Oil.

The results showed that the concentration of astaxanthin in the serum of ASTA and ASTA-krill oil group increased significantly after gavage, and the trend was similar. The serum content of the rats in the Antarctic krill oil group was low, and astaxanthin was not detected. In the ASTA-krill oil group, the astaxanthin concentration in the serum reached a high peak at $15.82 \pm 2.89 \mathrm{ug} / \mathrm{L}$ at $8 \mathrm{~h}$; in the subsequent $10-24$ $\mathrm{h}$, the concentration of astaxanthin in the serum gradually decreased to $16 \mathrm{~h}$. The concentration of astaxanthin in serum was $1.55 \pm 0.66 \mathrm{ug} / \mathrm{L}$. The ASTA-Fish oil group reached a high peak at $2.98 \pm 0.59 \mathrm{ug} / \mathrm{L}$ in $2 \mathrm{~h}$, followed by $4-24 \mathrm{~h}$, the concentration of astaxanthin in serum gradually decreased, and the concentration of astaxanthin in serum did not reach the limit of quantitation by $12 \mathrm{~h}$. not detected. Compared with the ASTA-Fish oil group, the concentration of astaxanthin in the serum of the rats fed the ASTA-krill oil group was delayed by $6 \mathrm{~h}$, and the highest concentration in the serum wasASTA- Fish oil group was 5.3 times, which was 8.4 times that of the ASTA group. The results of the study and Zhou Qingxin study the astaxanthin ester and free astaxanthin in mice, the same as the astaxanthin concentration-time curve in serum.

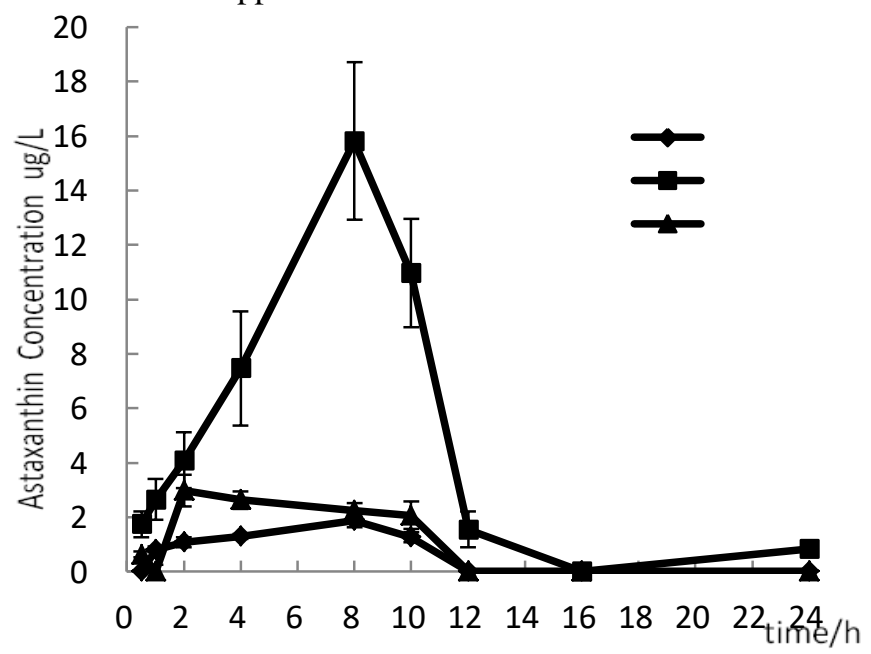

Fig. 3 Time profiles of Astaxanthin in the serum of Astaxanthin and Antarctic krill oil or fish oil in a pharmacokinetic study with a single oral administration. 
Table 4 The astaxanthin Concentration with single oral administration in the serum.

\begin{tabular}{ccccc}
\hline \multirow{2}{*}{ Time/h } & ASTA & ASTA-Krill Oil & $\begin{array}{c}\text { ASTA-Fish } \\
\text { Oil }\end{array}$ & $\begin{array}{c}\text { Antarctic } \\
\text { krill oil }\end{array}$ \\
\cline { 2 - 5 } & $\begin{array}{c}\mathrm{A} \\
(\mathrm{ug} / \mathrm{L})\end{array}$ & $\begin{array}{c}\mathrm{C} \\
(\mathrm{ug} / \mathrm{L})\end{array}$ & $\begin{array}{c}\mathrm{B} \\
(\mathrm{ug} / \mathrm{L})\end{array}$ & $\begin{array}{c}\mathrm{G} \\
(\mathrm{ug} / \mathrm{L})\end{array}$ \\
\hline 1.5 & 0.00 & $1.75 \pm 0.48$ & $0.63 \pm 0.10$ & 0.00 \\
2.0 & $0.82 \pm 0.11$ & $2.66 \pm 0.75$ & 0.00 & 0.00 \\
4.0 & $1.08 \pm 0.17$ & $4.09 \pm 1.03$ & $2.98 \pm 0.59$ & 0.00 \\
8.0 & $1.31 \pm 0.09$ & $7.47 \pm 2.10$ & $2.64 \pm 0.32$ & 0.00 \\
10.0 & $1.89 \pm 0.26$ & $15.82 \pm 2.89$ & $2.24 \pm 0.27$ & 0.00 \\
12.0 & $1.26 \pm 0.18$ & $10.96 \pm 1.98$ & $2.07 \pm 0.51$ & 0.00 \\
16.0 & 0.00 & $1.55 \pm 0.66$ & 0.00 & 0.00 \\
24.0 & 0.00 & 0.00 & 0.00 & 0.00 \\
$\mathrm{AUC} \mathrm{ug} \cdot \mathrm{h} / \mathrm{L}$ & $14.35 \pm 0.21$ & $108.39 \pm 2.34$ & $23.39 \pm 0.46$ & 0.00 \\
\hline
\end{tabular}

\section{Conclusion}

In this study, bioavailability of Astaxanthin, was compared with that of, ASTA-Fish Oil and ASTA-Krill Oil was found to have superior serum absorption and organ tissue accumulation rates for ASTA-krill oil, based on our in vivo studies. Consequently, Antarctic krill oil as functional food or special medical food,which improves astaxanthin bioavailability as demonstrated in this study.

\section{References}

1. Liu Li, Liu Chengchu, Zhao Yong et al. Nutritional health effects and food safety evaluation of Antarctic krill[J].Food Science,2010,31(17): 443-447.

2. Feng Dina, Yuan Ming, Zhu Xiaoli. Research Progress in Physiological Function of Antarctic Krill Oil[J]. Food Research and Development, 2015, 36(7): 115-117.

3. Zhang Xiaoli, Liu Jianguo. Antioxidation of Astaxanthin and Its Application in Nutrition and Medicine Applications[J].Food Science,2006,27(1):258-262.
4. Miao FP, Geng YH, Lu DY, et al. Stability and changes in astaxanthin ester composition from Haematococcus pluvialis during storage $[\mathrm{J}]$. Chinese Journal of Oceanology and Limnology , 2013 , 31( 6 ):1181 — 1189.

5. Wang Jiahui, Liu Chunhua, Ren Bingxing, Wang Yuming, Xue Changhu. Effects of different fatty acid composition phospholipids on digestion and absorption of rats $[\mathrm{J}]$, China Oils and Fats, 2013, 8:51-54.

6. Sun Weihong, Xing Lihong et al. Determination of astaxanthin in Antarctic krill and its products by high performance liquid chromatography[J] Journal of Food Safety and Quality, 2017.8(4).1248-1253

7. Hashimoto, T., Ozaki, Y., Taminato, M., Das, S. K., Mizuno, M., Yoshimura, KKanazawa, K. (2009). The distribution and accumulation of fucoxanthin and its metabolites after oral administration in mice. British Journal of Nutrition, 102(2), 242-248.

8. Sy, C., Gleize, B., Dangles, O., Landrier, J. F., Veyrat, C. C., \& Borel, P. (2012). Effects of physicochemical properties of carotenoids on their bioaccessibility, intestinal cell uptake, and blood and tissue concentrations. Molecular Nutrition \& Food Research,56(9), 1385-1397. 